\title{
UCRL-TR-202444
}

LAWRENCE LIVERMORE N A T IO N A L LABORATORY

\section{Applicability of the Spin-orbit Sum Rule for the Actinide 5? States}

G. van der Laan, K.T. Moore, J.G. Tobin, B.W. Chung, M.A. Wall, A.J. Schwartz

February 20, 2004 
This document was prepared as an account of work sponsored by an agency of the United States Government. Neither the United States Government nor the University of California nor any of their employees, makes any warranty, express or implied, or assumes any legal liability or responsibility for the accuracy, completeness, or usefulness of any information, apparatus, product, or process disclosed, or represents that its use would not infringe privately owned rights. Reference herein to any specific commercial product, process, or service by trade name, trademark, manufacturer, or otherwise, does not necessarily constitute or imply its endorsement, recommendation, or favoring by the United States Government or the University of California. The views and opinions of authors expressed herein do not necessarily state or reflect those of the United States Government or the University of California, and shall not be used for advertising or product endorsement purposes.

This work was performed under the auspices of the U.S. Department of Energy by University of California, Lawrence Livermore National Laboratory under Contract W-7405-Eng-48. 


\title{
Applicability of the spin-orbit sum rule for the actinide $5 f$ states
}

\author{
G. van der Laan, ${ }^{1}$ K. T. Moore, ${ }^{2}$ J. G. Tobin, ${ }^{2}$ \\ B. W. Chung, ${ }^{2}$ M. A. Wall, ${ }^{2}$ and A. J. Schwartz ${ }^{2}$ \\ ${ }^{1}$ Magnetic Spectroscopy, Daresbury Laboratory, \\ Warrington WA 4 \&AD, United Kingdom \\ ${ }^{2}$ Laurence Livermore National Laboratory, Livermore, California 94550, USA
}

(Dated: January 27, 2004)

\begin{abstract}
The branching ratio of core-valence transitions in $\mathrm{x}$-ray absorption spectroscopy and electron energy loss spectroscopy is linearly related to the expectation value of the spin-orbit operator of the valence states. Here, we analyse the measured branching ratio of the $N_{4,5}$ edges acquired by electron energy-loss spectroscopy in a transmission electron microscope, and synchrotron-radiationbased x-ray absorption. Results show that the spin-orbit sum rule can be applied to actinide $5 f$ states, where the accuracy can be increased using the correction term obtained from atomic manyelectron calculations.
\end{abstract}

PACS numbers: 78.70.Dm; 71.70.-d 71.10.-w; 71.27.+a. 
Spin-orbit coupling plays an integral role in the behavior of narrow-band materials. Nowhere is there as strong an example of this as in the actinides, where the $5 f$ bands are known to be narrow [1-3] and have a large spin-orbit interaction $[4,5]$, leading to behaviors, such as itinerant magnetism, heavy fermions, and superconductivity [6-10]. The spin-orbit interaction is also key to optical spin orientation and spin relaxation and makes the transport and spin phenomena interdependent The degree of spin-orbit interaction may be investigated using the sum rule for x-ray absorption, [11-14] potentially explaining the origin of the abovementioned behaviors observed in the $5 f$ states.

When an electron is excited from a core level to the valence band, the spectrum consists of transitions to two final-state manifolds which are well separated in energy by the spin-orbit interaction of the core hole. Transition metals, rare earths, and actinides exhibit this set of high-intensity peaks, often called white lines, at the threshold of absorption edges. The branching ratio of the white lines is directly related to the expectation value for the angular part of the spin-orbit operator of the valence states. [11-14] Using many-electron spectral calculations, where spin-orbit, Coulomb, and exchange interactions are treated on equal footing, it is possible to predict the core-level spectra. While it has been shown that the spin-orbit sum rule works well for $4 d$ and $5 d$ metals, the $3 d$ and $4 f$ metals are complicated by core-valence interactions. [12] In this Letter, we will show that the spin-orbit sum rule can be applied successfully to the actinides, using the $N_{4,5}(4 d \rightarrow 5 f)$ transition. We will apply this to $\mathrm{Th}, \mathrm{U}$ and $\mathrm{Pu}$ metal measured by both electron energy-loss spectroscopy (EELS) and $x$-ray absorption spectroscopy (XAS).

EELS experiments were performed using a Phillips CM300 field-emission-gun TEM, equipped with a Gatan imaging filter for the collection of spectra. Since the accelerating voltage of the TEM was $300 \mathrm{keV}$ and much larger than the energy of the $N_{4,5}$ transitions for $\mathrm{Th}, \mathrm{U}$, and $\mathrm{Pu}$, the electron transitions are justified as electric dipole [15]. EELS spectra were recorded in imaging mode using a collection angle of $6 \mathrm{mrad}$, which allowed the 000 beam and the first-order reflections to contribute to the spectra. All spectra were reproduced numerous times using a sample thickness of $\sim 0.5$ inelastic mean free path, as calculated by the zero-loss and plasmon peaks. Multiple spectra were recorded, aligned, and summed to optimize the signal-to-noise ratio and to maintain optimal energy resolution of $\sim 1 \mathrm{eV}$. Samples were $99.9 \%$ pure and were prepared via either electropolishing or ion milling. While Th and $\mathrm{U}$ could be handled directly, all $\mathrm{Pu}$ work was performed in a glove box, and the samples 
were transported in a vacuum-transfer holder due to the toxic nature of the metal (for full sample preparation procedures see [16]). The XAS spectra were collected at the Advanced Light Source (ALS) in total electron yield mode, using a $30 \mathrm{mg}$ sample of purified $\alpha$-Pu (details are provided elsewhere [17]).

The $N_{4,5}$ EELS edges for $\alpha$-Th, $\alpha$-U, and $\alpha$-Pu are shown in Fig. 1, each accompanied by a single-crystal diffraction pattern that confirms the phase being examined. An XAS spectrum from $\alpha$-Pu is also presented $[17,18]$. The spin-orbit split $4 d_{3 / 2}$ and $4 d_{5 / 2}$ manifolds are clearly resolved for each metal as a pair of white lines. No fine structure is resolved for either edge due to the intrinsic core-hole lifetime broadening of $\sim 2 \mathrm{eV}[14,19]$. Along the actinide series from $\mathrm{Th}$ to $\mathrm{Pu}$ the $4 d_{3 / 2}$ peak progressively reduces in intensity due to the fact that selection rules govern that a $d_{3 / 2}$ core electron can only be excited into an $f_{5 / 2}$ level [14].

For the $d \rightarrow f$ transitions, [20] the branching ratio is defined as $B \equiv A_{5 / 2} /\left(A_{5 / 2}+A_{3 / 2}\right)$, where $A_{5 / 2}$ and $A_{3 / 2}$ are the areas underneath the core level $d_{5 / 2}$ and $d_{3 / 2}$ peaks, respectively. The sum-rule [11-14] gives the relation

$$
\frac{\left\langle w^{110}\right\rangle}{n_{h}}=-\frac{5}{2}\left(B-B_{0}\right)
$$

where $\left\langle w^{110}\right\rangle$ is the expectation value of the $5 f$ spin-orbit electron-operator, $n_{h}$ is the number of $5 f$ holes, and $B_{0}$ is the value of the branching ratio without spin-orbit interaction in the $f$ shell. Similar to other XAS sum rules [21], the expectation value is obtained per hole, since the core electron is excited into empty valence states. The values per electron can be obtained by multiplying both sides of Eq. (1) by $n_{h} / n$.

In order to relate the results to physical quantities we need to know the precise meaning of $\left\langle w^{110}\right\rangle$. The spin-orbit interaction is given by a hamiltonian $(l \cdot s) \zeta_{l}$, where $l \cdot s$ and $\zeta_{l}$ are the angular and radial part, respectively, of the spin-orbit operator for the $l$ shell. The spin-orbit parameter $\zeta_{l}$ depends on the effective $Z$ (atomic) number, whereas the expectation value of the angular part $\langle l \cdot s\rangle=\frac{2}{3}\left\langle w^{110}\right\rangle$ (for the $f$ shell) depends on the electron distribution over the angular momentum sublevels, $j=l \pm 1 / 2$. It can be expressed in the electron occupation numbers $n_{7 / 2}$ and $n_{5 / 2}$ of the $j=7 / 2$ and $5 / 2$ levels,

$$
\left\langle w^{110}\right\rangle=n_{7 / 2}-\frac{4}{3} n_{5 / 2}
$$


with $n=n_{7 / 2}+n_{5 / 2}$ the total number of $f$ electrons. Thus $\left\langle w^{110}\right\rangle$ will be $-4 / 3$ for a $j=5 / 2$ electron and 1 for a $j=7 / 2$ electron; and $\left\langle w^{110}\right\rangle / n_{h}=0$ corresponds to the statistical distribution $3 n_{7 / 2}=4 n_{5 / 2}$. The spin-orbit operator can also be expressed as a hole operator (denoted by an underscore), where $\left\langle\underline{w}^{110}\right\rangle=-\left\langle w^{110}\right\rangle$. [14]

Equation (1) shows that in order to extract the value of $\left\langle w^{110}\right\rangle / n_{h}$ from the branching ratio, $B$, we need to know $B_{0}$. It has been shown that the value of $B_{0}$, which to first order is independent of the $5 f$ spin-orbit interaction, depends on the core-valence interaction [12]. If the latter interaction can be neglected, then $B_{0}$ is equal to the statistical value of $\frac{3}{5}$ (for a $d$ core hole) and Eq. (1) is exact. In general the spin-orbit-split core levels are mixed due to the core-valence interaction, so that the sum rule is not exact. This will result in a correction term $\Delta$, such that

$$
\frac{\left\langle w^{110}\right\rangle}{n_{h}}=-\frac{5}{2}\left(B-\frac{3}{5}\right)+\Delta
$$

where

$$
\Delta \equiv \frac{5}{2}\left(B_{0}-\frac{3}{5}\right)
$$

is related to the difference between $B_{0}$ and the statistical value. Using first-order perturbation theory, $\Delta$ is proportional to the ratio between the core-valence exchange interaction $G^{1}(c, l)$ and the energy splitting between the white lines, which is determined by the spinorbit parameter $\zeta_{c}$. Table I shows the remarkable proportionality between $G^{1}(c, l) / \zeta_{c}$ and $\Delta$ over a range of edges in $3 d, 4 d, 5 d, 4 f$, and $5 f$ metals. These numbers were obtained from relativistic atomic Hartree-Fock calculations using Cowan's code [22], where $B_{0}$ was calculated using the weighted average over the possible $J$ levels in the ground state.

From Table I we can make the following observations. For $3 d$ transition metals the application of the spin-orbit sum rule for the $L_{2,3}$ branching ratio is severely hampered by the large $(2 p, 3 d)$ exchange interaction which is of similar size as the $2 p$ spin-orbit interaction. [12] The same is true of the $M_{4,5}$ edges of the lanthanides, where the $(3 d, 4 f)$ exchange interaction is quite strong compared to the $3 d$ core spin-orbit interaction. [23] On the other hand, for the $L_{2,3}$ edges of $4 d$ and $5 d$ transition metals, associated with the deep $2 p$ core level, the sum rule is expected to hold quite well. The situation is also favorable for the actinides, where the calculated values of $G^{1}(c, 5 f) / \zeta_{c}$ for the $3 d$ and $4 d$ core levels as well as the correction term $\Delta$ for the associated $M_{4,5}$ and $N_{4,5}$ branching ratios are rather small. 
Here we will concentrate on the actinide $N_{4,5}$ edges. The values of $B$ are given in Table II, together with $\left\langle w^{110}\right\rangle / n_{h}$, and $\Delta$ for ground states with different $f^{n}$ configurations, calculated in intermediate coupling (IC) using Cowan's code [22]. The gradual decrease of $\Delta$ along the series is due to the increasing core spin-orbit splitting. [12] Furthermore, as long as $\Delta \ll\left\langle w^{110}\right\rangle / n_{h}$, the error in the sum rule will be small. The accuracy can be improved by using the calculated values of $\Delta$, which we will demonstrate below using the branching ratios of the $N_{4,5}$ edges measured by EELS and XAS.

Figure 2 shows a plot of $\left\langle w^{110}\right\rangle / n_{h}$ versus the number of $f$ electrons. In order to use the experimental values of $B$ they have to be converted using Eq. (3). The experimental values of $B$ for $\mathrm{Th}, \mathrm{U}$ and $\mathrm{Pu}$ metal are given in Table III together with the resulting values of $\left\langle w^{110}\right\rangle / n_{h}-\Delta$. The dotted lines give the curves for $\Delta=0$; the drawn lines give the curves where we used the calculated values of $\Delta$ for each $n$ as given in Table II. Those curves are useful if the number of $5 f$ electrons is unknown. The figure shows that the experimental uncertainty (typically the diffence between XAS and EELS) is slightly larger than the correction term $\Delta$.

Figure 2 also shows the theoretical curves for the three different coupling schemes, $j j$, $L S$ and IC. [14] For IC we used the relativistic atomic Hartree-Fock values from Table II. Equation (2) allows a straightforward interpretation of these curves in terms of the occupation numbers of the ground state $j$ levels. All expectation values are negative due to the predominant occupation of the $j=5 / 2$ level. In $j j$ coupling, the dominant $5 f$ spin-orbit interaction forces all electrons in the $j=5 / 2$ level so that $\left\langle w^{110}\right\rangle / n_{h}=-\frac{4}{3} n / n_{h}$ for $n \leq 6$. In $L S$ coupling the $5 f$ electrostatic interactions dominate over the $5 f$ spin-orbit interaction, so that $n_{7 / 2}$ is increased at the cost of $n_{5 / 2}$, thereby reducing the absolute value of $\left(w^{110}\right) / n_{h}$. In IC, the $5 f$ electrostatic interactions and the $5 f$ spin-orbit interaction compete with each other, so that $\left\langle w^{110}\right\rangle / n_{h}$ is between the limits of $j j$ and $L S$ coupling. It should be noted that $j j$ and $L S$ coupling are in fact only theoretical schemes, and that the atomic case has the IC value. In actinides the IC values are closer to the $j j$ coupling limit, as confirmed in Fig. 2, while in lanthanides they are closer to the $L S$ coupling limit, since the $4 f$ spin-orbit interaction is much smaller than the $5 f$. So called "quenching" of the spin-orbit interaction arises when the first-order term vanishes due to symmetry. Although in principle $\left\langle w^{110}\right\rangle$ could reduce to zero, in practice this seldomly happens due to non-vanishing higher-order terms. [24] 
With the aid of Fig. 2 we can now not only establish an allowed range for the number of $f$ electrons, but also make a connection between the number of $f$ electrons and the degree of quenching of the $5 f$ spin-orbit expectation value. Since the $\left\langle w^{110}\right\rangle / n_{h}$ value must be within the $j j$ coupling limit, we can infer from Fig. 2 that Th metal must have more than 0.75 $f$ electrons. Furthermore, for $\mathrm{U}$ metal to comply with IC (where $n_{7 / 2} / n \approx 2 \%$ ), it must have $n=2$. However, in order to satisfy $L S$ coupling (where $n_{7 / 2} / n \approx 25 \%$ ), $n$ would have to be close to 3 or above. Moreover, our experimental result shows that the spin-orbit interaction could not be significantly lower than the value for $L S$ coupling. For $\mathrm{Pu}$ the case is clear cut. Its $\left\langle w^{110}\right\rangle / n_{h}$ value is well outside the range of $L S$ coupling, and satisfies IC for $n=5$. Even if Pu would have $n=6$ the result is still closer to IC than to $L S$ coupling. This demonstrates that there are severe restrictions for the $f$ count when a specific choice of the spin-orbit coupling scheme is imposed. Therefore, the results obtained using the spin-orbit sum rule can provide important guidance for theoretical calculations. The case of the actinides is especially interesting because the spin-orbit interaction is very important in heavy atoms. In the literature, the precise number of $5 f$ electrons is sometimes controversial for these systems, where different calculational schemes are giving rather different $f$ counts. We also note that if we adopt the $f$ counts encountered most commonly in the literature, namely $f^{5}$ for $\mathrm{Pu}$ and $f^{3}$ for $\mathrm{U}$, this would mean IC for $\mathrm{Pu}$ but a considerable reduction from IC for $\mathrm{U}$. This coincides with the well known change in $5 f$ localization between the two metals.

In the actinides the outer $s$ and $d$ electron orbitals are broad and overlap strongly and therefore show a metallic behavior. Conversely, the $5 f$ orbitals which have a smaller radius have a more atomic like behavior. In the actinide series the lightest elements have metalliclike $f$ electrons, whereas the heavier actinides have atomic-like $f$ electrons. As the atomic number of the nucleus increases, the attractive electrostatic interaction increases, pulling the $f$ electrons closer to the atom. This causes the shape of the $f$ orbital to change from overlapping to non-overlapping. The localization will reduce the hybridization, crystal field interaction and, hence we expect a more atomic character, where $n_{5 / 2}$ is increased at the $\operatorname{cost}$ of $n_{7 / 2}$. This suggest that ultimately the branching ratio can be used as a probe of the localization.

This explains why band calculations such as unmodified density functional theory (DFT) work well with $\mathrm{U}$ [25], but fail for Pu unless augmented with the generalized gradient approx- 
imation with spin-orbit coupling [26]. The shutting off of the delocalization perturbation would explain much of the underlying cause of the recent success of sophisticated, but sometimes unorthodox, modeling of $\mathrm{Pu}$ electronic structure. These approaches include the ad hoc localization of the $5 f$ states [27], inclusion of magnetic polarization in both $\delta$-Pu $[1,28,29]$ and $\alpha-\mathrm{Pu}[30,31]$, a strongly relativistic, spin-orbit split model [32], and Kondo-type electron correlation [1]. It is also consistent with recent experimental work that reports localization effects in $\delta$-Pu [32]. Finally, the turning off of the delocalization perturbation occurs prior to the large volume change between $\alpha$-Pu and $\alpha$-Am, suggesting that electronic changes in the $5 f$ states are occurring well before the $\mathrm{Pu}$ volume anomaly.

In conclusion, our results show that the spin-orbit sum rule works excellently for the actinide metals. This means we now have an unambiguous probe for the $5 f$ spin-orbit interaction. The deviation caused by the core-valence exchange interaction is small and can be taken into account using a correction term. Application of sum rule yields accurate values of the spin-orbit interaction that can be used to restrict the range allowed for the $f$ count, thereby providing useful criteria for calculational schemes.

The key result here is that models that work well for U, e.g. DFT-type with delocalization and $L S$ coupling, must fail for $\mathrm{Pu}$. Between $\mathrm{U}$ and $\mathrm{Pu}$, the delocalization perturbation turns off or effectively becomes negligible. For $\mathrm{Pu}$, modeling which diminishes delocalization effects will be most appropriate. While some level of delocalization and concomitant hybridization with the $d$ states must be occurring in $\mathrm{Pu}[33], 5 f$ delocalization is a secondary effect relative to the $5 f$ spin-orbit splitting. Here, the spin-orbit sum rule clarified the coupling mechanisms and degree of $5 f$ localization quantitatively in $\mathrm{Th}, \mathrm{U}$, and $\mathrm{Pu}$, but the technique can also be used for other pressing questions, such as the degree of $5 f$ localization in the $\mathrm{Pu}$-based superconductor $\mathrm{PuCoGa}_{5}$ [8-10].

This work was partially performed under the auspices of the U.S. DOE by LLNL under contract W-7405-Eng-48. The ALS and Beamline 7.0 have been built and operated under funding from the Office of Basic Energy Science at DOE. The authors wish to thank J. Lashley and M. Blau for synthesis of the Pu samples and D. Shuh, R. Schulze, J. Terry, J. D. Farr, T. Zocco, K. Heinzelman, and E. Rotenberg for help with the data collection.

[1] S. Y. Savrasov and G. Kotliar, Phys. Rev. Lett. 84, 3670 (2000). 
[2] S. Y. Savrasov, G. Kotliar, and E. Abrahams, Nature (London) 410, 793 (2001).

[3] S. S. Hecker, MRS Bulletin 26, 672 (2001) and references therein.

[4] A. B Shick and W. E. Pickett, Phys. Rev. Lett. 86, 300 (2001).

[5] K. T. Moore, M. A. Wall, A. J. Schwartz, B. W. Chung, D. K. Shuh, R. K. Schulze, and J. G. Tobin, Phys. Rev. Lett. 90, 196404 (2003).

[6] D. L. Cox, Phys. Rev. Lett. 59, 1240 (1987).

[7] D. L. Cox and M. B. Maple, Phys. Today 48, 32 (1995).

[8] J. L. Sarrao, L. A. Morales, J. D. Thompson, B. L. Scott, G. R. Stewart, F. Wastin, J. Rebizant, P. Boulet, E. Colineau, and G. H Lander, Nature (London) 420, 297 (2002).

[9] I. Opahle and P. M. Oppeneer, Phys. Rev. Lett. 90, 157001 (2003).

[10] T. Maehira, T. Hotta, K. Ueda, and A. Hasegawa, Phys. Rev. Lett. 90, 207007 (2003).

[11] G. van der Laan and B. T. Thole, Phys. Rev. Lett. 60, 1977 (1988).

[12] B. T. Thole and G. van der Laan, Phys. Rev. B 38, 3158 (1988).

[13] B. T. Thole and G. van der Laan, Phys. Rev. A 38, 1943 (1988).

[14] G. van der Laan and B. T. Thole, Phys. Rev. B 53, 14458 (1996).

[15] R. F. Egerton, Electron energy-loss spectroscopy in the electron microscope, 2nd ed. (Plenum Press, NY, 1996) p. 221.

[16] K. T. Moore, M. A. Wall and A. J. Schwartz, J. Nucl. Mater. 306, 213 (2002).

[17] J. G. Tobin, B. W. Chung, R. K. Schulze, J. Terry, J. D. Farr, D. K. Shuh, K. Heinzelman, E. Rotenberg, G. D. Waddill, and G. van der Laan, Phys. Rev. B 68, 155109 (2003).

[18] In the raw XAS data it was possible to see the inverted Ni $2 p$ white lines that were superimposed on the Pu $4 d$ edge structure, due to a reduction of the $I_{0}$ beam by $\mathrm{x}$-ray absorption in some part of the beamline optics. This $\mathrm{Ni}$ spectral interference was removed prior to branching ratio determination.

[19] G. Kalkowski, G. Kaindl, W. D. Brewer, and W. Krone, Phys. Rev. B 35, 2667 (1987).

[20] For $p \rightarrow d$ transitions the relevant equations are $B=A_{3 / 2} /\left(A_{3 / 2}+A_{1 / 2}\right) ;\left\langle w^{110}\right\rangle / n_{h}=$ $-3\left(B-B_{0}\right)=-3\left(B-\frac{2}{3}\right)+\Delta ;\langle l \cdot s\rangle=\left\langle w^{110}\right\rangle=n_{5 / 2}-\frac{3}{2} n_{3 / 2} ; B_{0}=\frac{2}{3} ; \Delta \equiv 3\left(B_{0}-\frac{2}{3}\right)$.

[21] B. T. Thole, P. Carra, F. Sette, and G. van der Laan, Phys. Rev. Lett. 68, 1943 (1992).

[22] R. D. Cowan, The Theory of Atomic Structure and Spectra (University of California Press, Berkeley, 1981).

[23] B. T. Thole, G. van der Laan, J. C. Fuggle, G. A. Sawatzky, R. C. Karnatak, and J. M. 
Esteva, Phys. Rev. B 32, 5107 (1985).

[24] G. van der Laan, J. Phys.: Condens. Matter 10, 3239 (1998).

[25] P. Söderlind, Adv. Phys. 47, 959 (1998).

[26] P. Söderlind, Europhys. Lett. 55, 525 (2001).

[27] J. M. Wills , O. Eriksson, A. Delin, P. H. Andersson, J.J. Joyce, T. Durakiewicz, M. T. Butterfield, L. A. Morales, D. P. Moore, and A. J. Arko, Cond-Mat/0307767 (2003).

[28] P. Söderlind, A. Landa, and B. Sadigh, Phys. Rev. B 66, 205109 (2002).

[29] A. L. Kutepov and S. G. Kutepova, J. Phys.: Condens. Matter 15, 2607 (2003).

[30] B. Sadigh et al., Phys. Rev. B XX, XXXXX (2004).

[31] M. Penicaud, J. Phys.: Condens. Matter 9, 6341 (1997).

[32] L. Havela, T. Gouder, F. Wastin, and J. Rebizant, Phys. Rev. B 65, 235118 (2002).

[33] B. R. Cooper, R. Sieman, D. Yang, P. Thayamballi, and A. Banerjea, in Handbook of the Physics and Chemistry of the Actinides Eds. A. J. Freeman and G. H. Lander, Vol. 2 (Elsevier Science, Amsterdam, 1985).

FIG. 1: The $N_{4,5}(4 d \rightarrow 5 f)$ edges from $\alpha$-Th, $\alpha$-U, and $\alpha$-Pu acquired by EELS in a TEM and from $\alpha$-Pu acquired by XAS. A single-crystal diffraction pattern from each metal is presented, confirming the phase being examined by EELS.

FIG. 2: The spin-orbit expectation value per hole versus the number of $5 f$ electrons $(n)$. The experimental values are given by $\left\langle w^{110}\right\rangle / n_{h}=-\frac{5}{2}\left(B-\frac{3}{5}\right)+\Delta$ for $\mathrm{Th}, \alpha$-U and $\alpha$-Pu (see Table III) with $\Delta=0$ (thin horizontal lines) and using $\Delta$ for each $n$ from Table II (thick lines), (E = EELS and $\mathrm{X}=\mathrm{XAS}$ ). Also plotted are the theoretical curves for the different coupling schemes; $L S$ (short dash), $j j$ (long dash), and intermediate coupling (IC) (solid). 
TABLE I: The calculated values for $G^{1}(c, l) / \zeta_{c}$ and the correction term $\Delta=\frac{5}{2}\left(B_{0}-\frac{3}{5}\right)$ for a range of different absorption edges in $3 d, 4 d$ and $5 d$ transition metal, lanthanide and actinides. The linear relation between both quantities is evident from the numbers.

\begin{tabular}{cccc}
\hline & $c \rightarrow l$ & $G^{1}(c, l) / \zeta_{c}$ & $\Delta$ \\
\hline Ti $3 d^{0}$ & $L_{2,3}(2 p \rightarrow 3 d)$ & 0.981 & -0.895 \\
La $4 f^{0}$ & $M_{4,5}(3 d \rightarrow 4 f)$ & 0.557 & -0.485 \\
$\operatorname{Th} 5 f^{0}$ & $N_{4,5}(4 d \rightarrow 4 f)$ & 0.041 & -0.020 \\
$\operatorname{Th~} 5 f^{0}$ & $M_{4,5}(3 d \rightarrow 5 f)$ & 0.021 & -0.018 \\
Zr $4 d^{0}$ & $L_{2,3}(2 p \rightarrow 4 d)$ & 0.018 & -0.015 \\
Hf 5d & $L_{2,3}(2 p \rightarrow 5 d)$ & 0.002 & -0.002 \\
\hline
\end{tabular}

TABLE II: Calculations in intermediate coupling (IC) for different $5 f^{n}$ configurations. The branching ratio, $B$, of the $N_{4,5}$ edge spectra; the expectation value of the $5 f$ spin-orbit interaction per hole; $\left\langle w^{110}\right\rangle / n_{h}$; the branching ratio $B_{0}$ without $5 f$ spin-orbit interaction; and the resulting $\Delta=\frac{5}{2}\left(B_{0}-\frac{3}{5}\right)$.

\begin{tabular}{ccccc}
\hline$n$ & $B$ & $\left\langle w^{110}\right\rangle / n_{h}$ & $B_{0}$ & $\Delta$ \\
\hline 0 & 0.592 & 0 & 0.592 & -0.020 \\
1 & 0.634 & -0.1026 & 0.593 & -0.017 \\
2 & 0.680 & -0.2145 & 0.594 & -0.014 \\
3 & 0.723 & -0.3184 & 0.596 & -0.010 \\
4 & 0.760 & -0.4043 & 0.598 & -0.005 \\
5 & 0.817 & -0.5419 & 0.600 & 0 \\
6 & 0.918 & -0.7888 & 0.602 & 0.005 \\
\hline
\end{tabular}


TABLE III: Experimental values for the branching ratio, $B$, of the $N_{4,5}$ edge spectra of different actinides, and the resulting value of $\left\langle w^{110}\right\rangle / n_{h}-\Delta=\frac{5}{2}\left(B-\frac{3}{5}\right)$.

\begin{tabular}{ccc}
\hline & $B$ & $\left\langle w^{110}\right\rangle / n_{h}-\Delta$ \\
\hline $\operatorname{Th}(\mathrm{EELS})^{a}$ & 0.623 & -0.058 \\
$\alpha-\mathrm{U}(\mathrm{XAS})^{b}$ & 0.676 & -0.190 \\
$\alpha-\mathrm{U}(\mathrm{EELS})^{a}$ & 0.685 & -0.213 \\
$\alpha-\mathrm{Pu}(\mathrm{XAS})^{c}$ & 0.813 & -0.533 \\
$\alpha-\mathrm{Pu}(\mathrm{EELS})^{a}$ & 0.826 & -0.565 \\
\hline
\end{tabular}

aThis work (c.f., Fig. 1).

${ }^{b}$ Kalkowski et al., Ref. 19.

'Tobin et al., Ref. 17. 


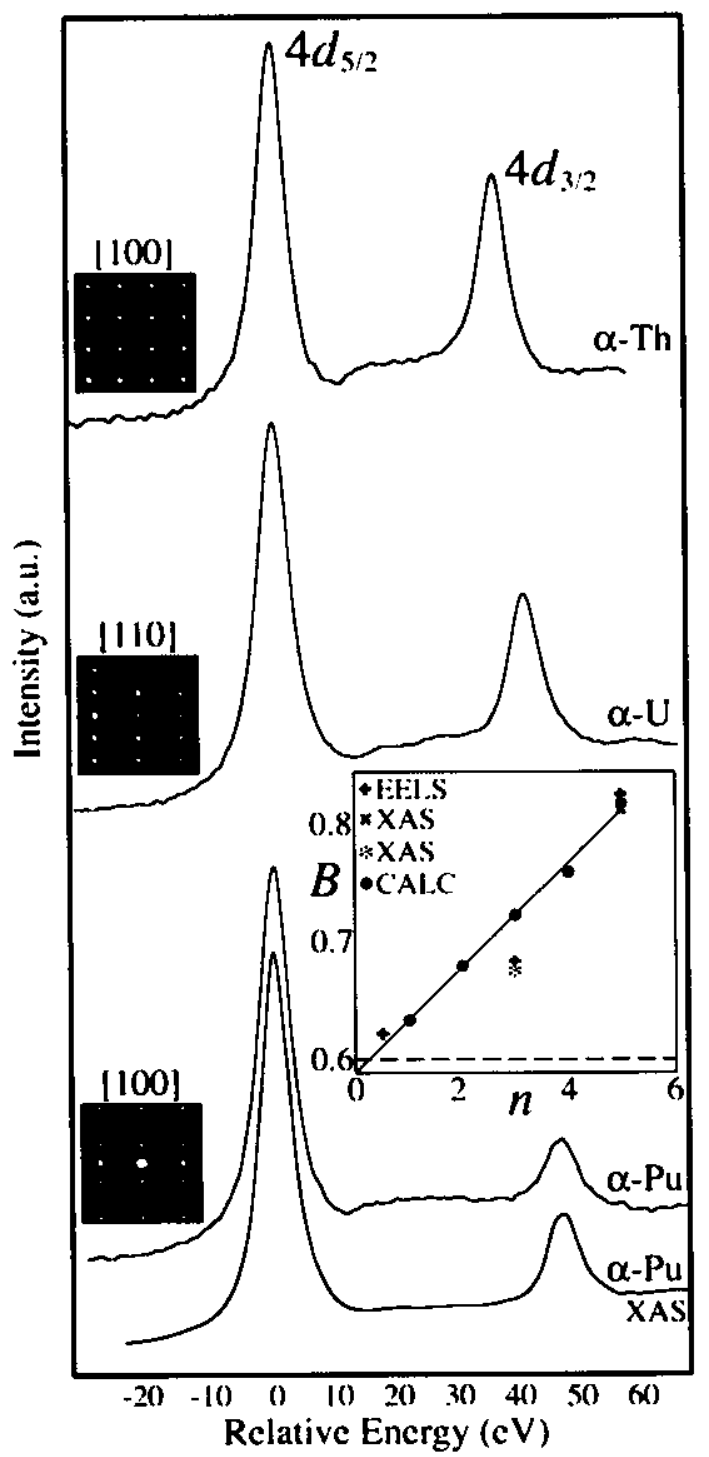

Figure 1 


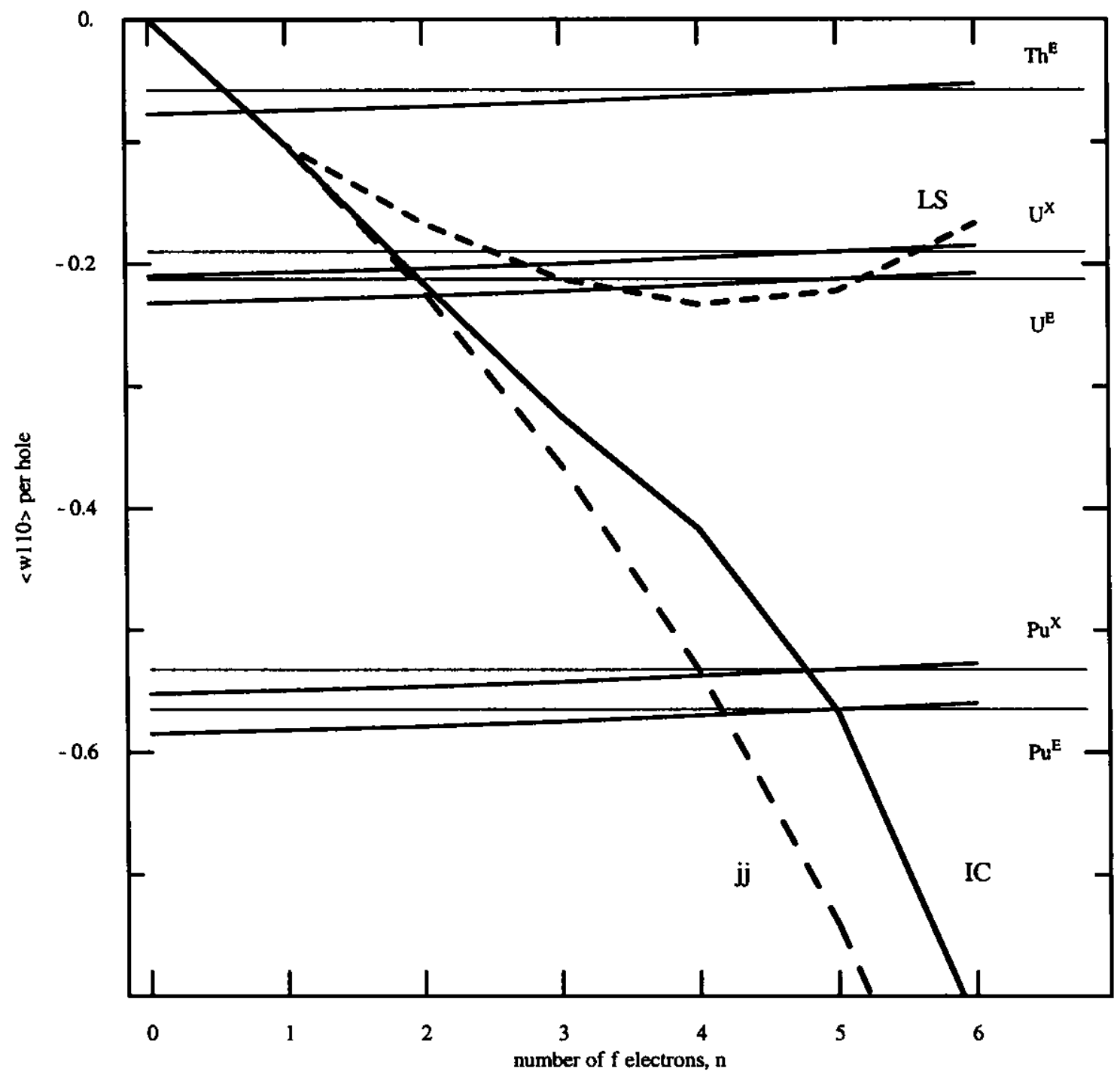

